

\title{
Junta Directiva Nacional 2012-2014
}

\section{Presidente}

Natalia Londoño Palacio

Secretario Ejecutivo

Natalia Malaver

\section{Tesorero}

Hector Fabio Sánchez Galvez

Fiscal

Alvaro Enrique Granados Díaz

Fiscal Suplente

Harold Aníbal Miranda Rosero

Presidente Electo 2014-2016

Helí Hernandez Ayazo

\section{Presidentes Capítulos}

Alto Magdalena

Maria del Pilar Peña Tovar

Antioquia-Chocó

Juan Fernando Velásquez Osorio

Caldas

Fabio Mauricio Sánchez Cano

Caribe

Edward Torres Gómez

\section{Central}

Walter Hernando Villalobos Monsalve

Costa Atlántica

Angelo María Alario Bello

Morrosquillo

Máximo Rafael Mercado Vergara

Nariño

Álvaro Portilla Cabrera

Norte de Santander

Juan Carlos Ortega Madariaga

Quindío

José Javier Arango Álvarez

Risaralda

Alex Arnulfo Rivera Toquica

Santander

Gustavo Adolfo Parra Zuluaga

Valle - Cauca

Gilberto Castillo Barrios
Ex presidentes

Rafael Carrizosa ${ }^{+}$

Roberto De Zubiría +

Gustavo Montejo ${ }^{+}$

Adolfo De Francisco

William Rojas

Eduardo De Zubiría

Hernando Sarasti

Roso Alfredo Cala ${ }^{+}$

Fernando Chalem ${ }^{+}$

Pérez Starusta

José María Mora +

Alberto Restrepo ${ }^{+}$

Roberto Esguerra

Jaime Campos ${ }^{+}$

Darío Maldonado

Javier Molina

Hernando Matiz

Adolfo Vera

Roberto D'Achiardi

Guillermo Vanegas

Dora Inés Molina de Salazar

Gustavo Márquez

María Nelly Niño de Arboleda

Jairo Roa

Gregorio Sánchez

Juan Manuel Senior 


\section{Anuncios ACMI}

\section{Congreso Colombiano de Medicina Interna "Medicina humana e integral en Medicina Interna"}

\section{7-10 de agosto de 2014}

Centro de Convenciones, Cartagena de Indias

\section{Trabajos de investigación}

Fecha límite para recepción de resúmenes:

15 de abril de 2014

Fecha respuesta de aceptación de resúmenes:

30 de abril 2014

\section{Modalidades}

I. Texto completo

- Trabajo de investigación clínica o básica en medicina del adulto

- Trabajo de investigación realizada por residente.

II. Póster electrónico

Consulte requisitos específicos de presentación para cada modalidad: www.congresosapmi.com $\sim$ www.acmi.org.co

\begin{tabular}{|c|c|}
\hline \multicolumn{2}{|l|}{ Inscripciones } \\
\hline Categoría & Valor (hasta el 10 de agosto) \\
\hline Miembros ACMI a paz y salvo & Sin costo \\
\hline Médicos especialistas & $\$ 400.000$ \\
\hline Médicos generales & $\$ 350.000$ \\
\hline Estudiantes de medicina y otros profesionales (con acreditación & $\$ 300.000$ \\
\hline $\begin{array}{l}\text { La inscripción incluye: } \\
\text { - Acceso a todas las sesiones científicas } \\
\text { - Acceso a los cursos precongreso: jueves } 07 \text { de agosto } \\
\text { - Material: maletín, programa oficial, agenda y esfero }\end{array}$ & $\begin{array}{l}\text { Acceso a la muestra comercial } \\
\text { Ingreso a actividad de inauguración } \\
\text { Certificado de asistencia on-line }\end{array}$ \\
\hline
\end{tabular}

\section{Informes}

- Asociación Procongreso de Medicina Interna Cra. 16 A No. 77-11 - Of. $204 \sim$ Telefax (1) 5303435 - $5303383 \sim$ Bogotá, D.C. apmi2@etb.net.co www.congresos@apmi.com

- Asociación Colombiana de Medicina Interna www.acmi.org.co

\section{Congreso Mundial de Medicina Interna "Medicina Interna y más allá: hacia un mundo más saludable"}

\section{4-28 de octubre de $2014 \cdot$ Seul, Corea}

Informes: UCIM 2014 - http://www.wcim2014.org

\section{Secretariat for the WCIM 2014}

Address : 9th Fl., Samick Lavied'or Bldg., 234, Teheran-ro, Gangnam-gu, Seoul 135-920, Korea Tel : +82-2-566-2229, 566-2246 Fax : +82-2-6254-8049 E-mail: wcim2014@intercom.co.kr 